\title{
A Multiple Local Models Approach to Accuracy Improvement in Continuous Glucose Monitoring
}

\author{
Fátima Barceló-Rico, M.Sc., Jorge Bondia, Ph.D., José Luis Díez, Ph.D., and Paolo Rossetti, Ph.D.
}

\begin{abstract}
Background: Continuous glucose monitoring (CGM) devices estimate plasma glucose (PG) from measurements in compartments alternative to blood. The accuracy of currently available CGM is yet unsatisfactory and may depend on the implemented calibration algorithms, which do not compensate adequately for the differences of glucose dynamics between the compartments. Here we propose and validate an innovative calibration algorithm for the improvement of CGM performance.

Methods: CGM data from GlucoDay ${ }^{\circledR}$ (A. Menarini, Florence, Italy) and paired reference PG have been obtained from eight subjects without diabetes during eu-, hypo-, and hyperglycemic hyperinsulinemic clamps. A calibration algorithm based on a dynamic global model (GM) of the relationship between PG and CGM in the interstitial space has been obtained. The GM is composed by independent local models (LMs) weighted and added. LMs are defined by a combination of inputs from the CGM and by a validity function, so that each LM represents to a variable extent a different metabolic condition and/or sensor-subject interaction. The inputs best suited for glucose estimation were the sensor current $I$ and glucose estimations $\hat{G}$, at different time instants $\left[I_{k}, I_{k-1}\right.$, $\left.\hat{G}_{k-1}\right](I I G)$. In addition to $I I G$, other inputs have been used to obtain the GM, achieving different configurations of the calibration algorithm.

Results: Even in its simplest configuration considering only IIG, the new calibration algorithm improved the accuracy of the estimations compared with the manufacturer's estimate: mean absolute relative difference $(\mathrm{MARD})=10.8 \pm 1.5 \%$ versus $14.7 \pm 5.4 \%$, respectively $(P=0.012$, by analysis of variance). When additional exogenous signals were considered, the MARD improved further $(7.8 \pm 2.6 \%, P<0.05)$.

Conclusions: The LM technique allows for the identification of intercompartmental glucose dynamics. Inclusion of these dynamics into the calibration algorithm improves the accuracy of PG estimations.
\end{abstract}

\section{Introduction}

$S^{n}$ ELF-MONITORING OF BLOOD GLUCOSE is recognized as a milestone of diabetes care since the 1980s, allowing for improvement of metabolic control, especially in insulintreated patients. ${ }^{1}$ This has prompted research in the field of continuous glucose monitoring (CGM) with a growing body of evidence, although not definite, that it might translate into better glycemic control compared with selfmonitoring of blood glucose. ${ }^{2}$ However, the accuracy of currently available CGM devices is relatively poor, especially in the hypoglycemic range, where the false-positive and false-negative rates are unacceptably high. ${ }^{3-5} \mathrm{Al}-$ though small improvements have been achieved with the newest sensor generations, ${ }^{6,7}$ this is a critical issue because accurate prediction of plasma glucose (PG) is strongly needed in diabetes subjects before these systems are largely used either for glucose monitoring only or with the aim of "closing the loop" for a regulated insulin infusion by a minipump in the future.

Current commercially available CGM systems for home monitoring are subcutaneous electrochemical sensors. All of them use the glucose oxidase enzyme-based technology, which give a measure of glucose concentration into the interstitial fluid in terms of intensity of the current generated from the enzymatic reaction (expressed in nA). ${ }^{8}$ This means that a CGM system actually needs calibration against concurrent blood glucose values, thus providing an estimate of blood glucose concentration.

Actually, the current intensity from subcutaneous glucose sensors is the result not only of the interstitial glucose concentration, but also of the complex interaction between the sensor and the subject. Therefore the accuracy of the estimation of blood glucose from the measurement in the interstitial fluid will depend, among other factors, on the calibration algorithm. Indeed, the latter is a function that includes the relationship

University Institute of Control and Industrial Informatics, Polytechnical University of Valencia, Valencia, Spain. 
between PG and the sensor signal from interstitial fluid glucose, trying to minimize physiological differences between the two compartments. Unfortunately, however, few studies have systematically investigated this relationship, ${ }^{9-14}$ with heterogeneous results. This highlights the complexity of the PG-tointerstitial glucose dynamics and of the subject-to-sensor interaction, which contrasts with the rather simplistic approach of calibration algorithms currently implemented in commercial CGM systems ${ }^{15}$ (basically linear regression methods).

Indeed, linear regression models implemented in a CGM system usually require calibration under conditions of relative glycemic stability (at "stationary" metabolic states) where equilibrium between PG and interstitial glucose is expected. In this way, the relationship between the measured current intensity and PG is considered static, and both the plasma to interstitium transport dynamics and the possibly subjectspecific sensor interaction are neglected. This has been recognized as a limitation of currently calibration algorithms, but only a few authors have proposed alternative calibrations strategies, ${ }^{16-18}$ each one with some practical limitations that prevent its application to clinical practice. ${ }^{19}$

An alternative approach is to consider that the relationship between PG and the sensor signal from interstitial glucose is not linear and likely depends on the metabolic status. In this case, accurate estimation of PG requires mathematical models describing the relationship between PG and the electrical signal generated by interstitial fluid glucose concentrations, during both steady-state and dynamic conditions. ${ }^{16-18}$

The aim of this article is to describe a new calibration algorithm based on the integration of several local dynamic models, each one valid in a specific region, to estimate the PG level from measurements in a remote compartment (in this case, the interstitial space). The use of multiple local models (LMs) allows for a better description of the complexity of the PG to interstitial glucose dynamics, improving the accuracy of the estimations. Suitability and performance of this new technique are tested with a data set from a clinical study, where the sensor's current signal, glucose estimates, and paired reference PG measurement are available. Results are expressed in terms of accuracy of glucose estimates from the new calibration algorithm and compared with those obtained with the standard calibration procedure.

\section{Methods}

The proposed algorithm ${ }^{20}$ has been developed and validated using data from eu-, hypo-, and hyperglycemic clamps, where healthy subjects wore a microdialysis-based glucose sensor (GlucoDay ${ }^{\circledR}$, A. Menarini, Florence, Italy). The study has been described in detail elsewhere. ${ }^{21}$ In brief, after an initial period of spontaneous euglycemia, at time $+30 \mathrm{~min}$ an insulin infusion was started, at the rate of $1 \mathrm{mU} / \mathrm{kg} / \mathrm{min}$, and continued until $120 \mathrm{~min}$. At the same time, glucose (20\% [wt/ vol] dextrose) was infused when necessary at a variable rate allowing for a controlled slow fall of PG in about $60 \mathrm{~min}$, until the target of approximately $50 \mathrm{mg} / \mathrm{dL}$ was reached. From time $+90 \mathrm{~min}$ to $+120 \mathrm{~min}$ PG was maintained at a hypoglycemic plateau of approximately $50 \mathrm{mg} / \mathrm{dL}$. At time +120 min insulin infusion was stopped, and the glucose infusion rate was increased to raise $\mathrm{PG}$ after $45 \mathrm{~min}$ to the target of approximately $165 \mathrm{mg} / \mathrm{dL}$ (time $+165 \mathrm{~min}$ ), which was maintained for the next $15 \mathrm{~min}$ until time $+180 \mathrm{~min}$. PG was measured every 6 min synchronously with the output of the sensor, which gave a glucose estimate every $3 \mathrm{~min}$.

\section{Principles of the new calibration algorithm}

The algorithm is based on a model that describes the relationship between PG concentrations and the signal from the sensor. The structure of the model is described in detail elsewhere. ${ }^{22}$ Basically, it is based on the integration of several LMs (Fig. 1), each one characterized by independency and regional validity, in order to obtain an interpretable global model (GM):

$$
G M=\sum_{i=1}^{c} W L M_{i}
$$

where $W L M_{i}$ is the weighted LM $i$. Each weighted LM, $W L M_{i}$, is the result of the $L M L M_{i}$ multiplied by its validity function $V_{i}$ :

$$
W L M_{i}=V_{i} \cdot L M_{i}
$$

The structure of each LM could be any, but in this calibration algorithm linear models are used to add interpretability to the GM:

$$
L M_{i}=\beta_{1 i} \cdot x_{1}+\beta_{2 i} \cdot x_{2}+\ldots+\beta_{D i} \cdot x_{D}+\beta_{0 i}=\beta_{1 D_{i}} \cdot x_{1 D}+\beta_{0 i}
$$

where $x_{1 D}$ is the $D$-dimensional vector of inputs of the system and $\left[\beta_{1 D}, \beta_{0 i}\right]$ is the $D+1$-dimensional vector of the regression coefficients $\beta$ of each linear LM with $\beta_{0}$ an independent term.

As represented in Eq. 2, each LM is weighted by some validity functions $\left(V_{i}\right)$ that, in the proposed algorithm, are chosen to be hyper-Gaussian functions. ${ }^{23}$ They are normalized in order to define the degree of validity of the $i^{\text {th }}$ LM in the input's space so that $V_{i}=1$ if the $i^{\text {th }} \mathrm{LM}$ is $100 \%$ representative of the current input; in contrast, $V_{i}=0$ if the $i^{\text {th }}$ LM does not characterize the current input at all. Equations 4 and 5 show the final normalized hyper-Gaussian validity function for the one-dimensional and multidimensional cases, respectively:

$$
\begin{gathered}
V_{i}=e^{-\frac{1}{2}\left(\left(\frac{x-m_{i}}{\sigma_{i}}\right)^{2}\right)^{H}} \\
V_{i}=e^{-\frac{1}{2}\left(\left(\vec{x}-\vec{m}_{i}\right)^{T} \Sigma_{i}^{-1}\left(\vec{x}-\vec{m}_{i}\right)\right)^{H}}
\end{gathered}
$$

where $x$ is the input and $m_{i}$ and $\sigma_{i}$ are the center and the SD, respectively, of the $i^{\text {th }}$ validity function. For the multidimensional case, $\vec{x}$ and $\vec{m}$ are vectors, $T$ refers to the transposed vector, and ${ }_{\Sigma}$ is the diagonal matrix of variances of each validity function:

$$
\Sigma=\left[\begin{array}{llll}
\sigma_{1}^{2} & 0 & 0 & 0 \\
0 & \sigma_{2}^{2} & 0 & 0 \\
& & \ddots & \\
0 & 0 & 0 & \sigma_{D}^{2}
\end{array}\right]
$$

The use of hyper-Gaussian functions allows the algorithm to find compact regions where an LM is valid. Moreover, the number of parameters to define the model is reduced because only two parameters have to be defined for each input $x_{j}$ and each LM: mean $\left(m_{i j}\right)$ and variance $\left(\sigma_{i j}^{2}\right)$. Validity functions are independent functions, making each LM independent as well. This facilitates finding LMs defined by different characteristics 


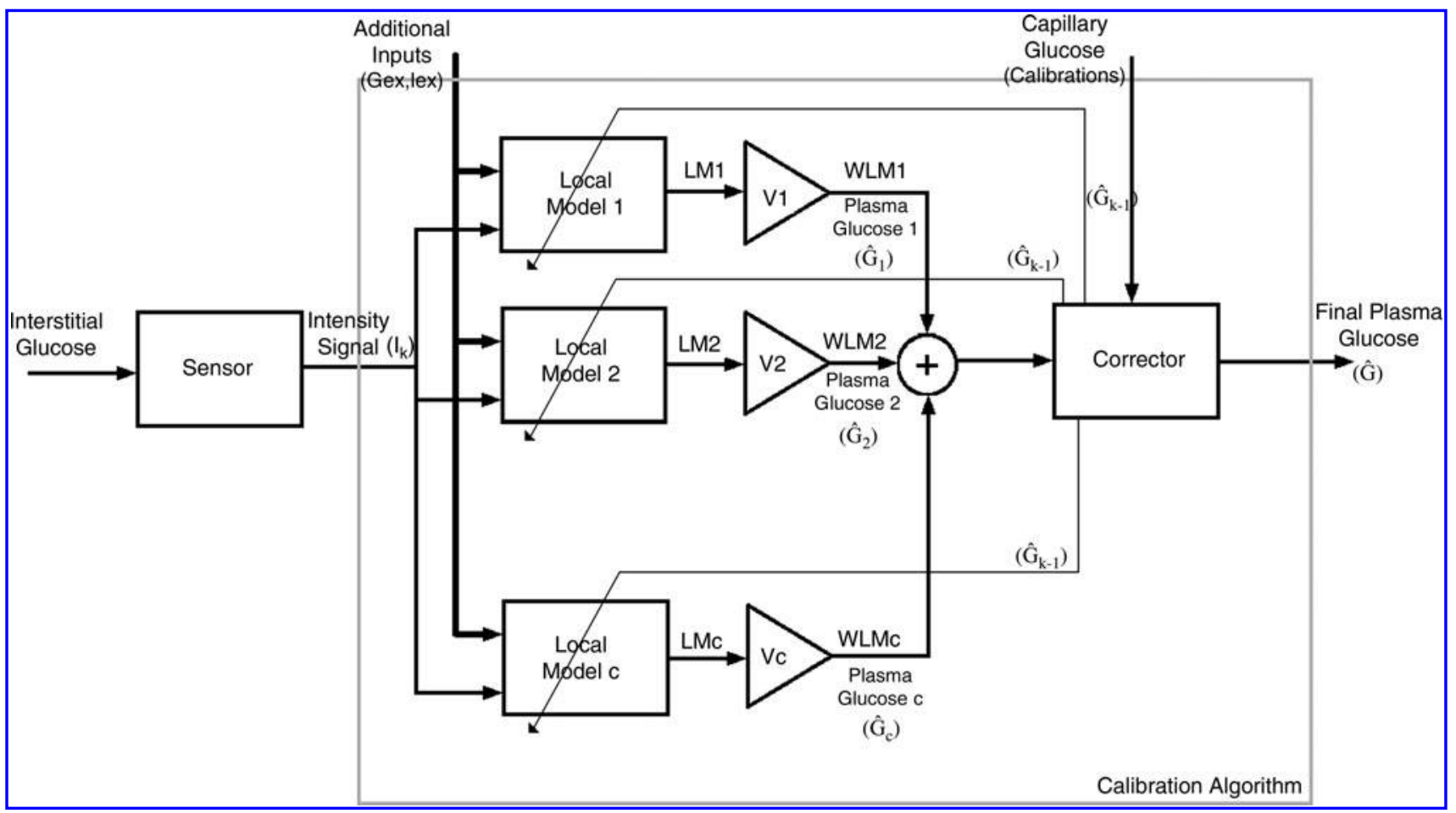

FIG. 1. Inputs from a remote compartment, in this case the interstitial space, feed the calibration algorithm. The basic inputs include $I_{k}, I_{k-1}$, and $\hat{G}_{k-1}$ (current and past intensity value from the sensor and past glucose estimation, respectively) and define both which local models (LMs) and to what extent they are valid. The latter is achieved weighing each LM by a validity function $V_{i}$ and minimizing both local and global errors. Weighed LMs (WLMs) are added, resulting in the global model, which provides the global glucose estimation. A block to consider the calibration point(s) from capillary measurements is included to correct the estimation of the algorithm. If available, additional signals can also be included (presence/absence of insulin or glucose infusion, meal, exercise, etc.).

of the inputs (i.e., different dynamics representative of the relationship between interstitial glucose and PG influenced by the interaction sensor/subject).

In summary, the resulting model will be described by a set of parameters: the regression coefficients of the linear models and the means and variances of the hyper-Gaussian membership functions, all of them defined for each LM. The final structure of the model can be seen in Eq. 6, already adapted for the $D$-dimensional case:

$$
G M=\sum_{i=1}^{c}\left(e^{-\frac{1}{2}\left(\left(\vec{x}-\vec{m}_{i}\right)^{T} \Sigma_{i}^{-1}\left(\vec{x}-\vec{m}_{i}\right)\right)^{H}} \cdot\left(\beta_{1 D_{i}} \cdot x+\beta_{0 i}\right)\right)
$$

The total number of parameters to identify will only depend on the number of inputs considered and on the number of LMs.

An additional feature of the proposed algorithm is that it is structured to minimize both the error of the glucose estimation from each LM (the difference between each LM's glucose estimation and reference glucose [local error]) and the error of the GM (the difference between the GM's glucose estimation and reference glucose [global error]). Optimal LM and validity function parameters are obtained through global optimization of a cost index comprising global and local errors. However, greater importance is given to the accuracy of glucose estimation from each LM compared with the accuracy of the GM. ${ }^{21}$ This strengthens the ability of the algorithm to find LM valid regionally, with each one characterizing to a different degree ( $0 \%$ to $100 \%$ ) different PG to interstitial glucose dynamics and/or sensor-subject interactions.
Finally, it is worth mentioning that, as the calibration algorithm may use inputs of different nature, normalization is required to make them all have, a priori, the same weight in the modeling process.

Glucose estimation given by the model is corrected every time a new calibration point is introduced, updating past values.

\section{Modeling the relationship between $P G$ and the sensor's electrical signal}

What follows is a description of the application of the above methodology to the available clinical data.

The processes of finding the best inputs that define the output (i.e., PG) and the proper number of LMs that compose the GM are dependent. Indeed, to avoid complexity, the aim is to find the minimum number of LMs that best define the output, and for that the input signals that contain more information about the output are needed.

Several combinations of inputs (samples of current [I], PG or capillary glucose $[G]$, and glucose estimations $[\hat{G}])$ in different time instants $(k-1, k-2, k-n)$ may be used to compute an estimation of the current PG level. However, with the available database, we found that the combination of inputs that best predicted the output was $\left[I_{k}, I_{k-1}, \hat{G}_{k-1}\right]$ (abbreviated by IIG). This means that the estimation of the actual glucose concentration at time $k, \hat{G}_{k}$, depends on the current intensity in the same time instant and involves a derivative indicating the trend of the signal. Using this input structure only two linear LMs $(c=2)$ 
were needed to achieve a good estimation of PG. Thus, the GM obtained in this case has the structure reported in Eq. 6, with

$$
\vec{x}=\left[\begin{array}{lll}
I_{k} & I_{k-1} & \hat{G}_{k-1}
\end{array}\right]
$$

and $c=2$.

Of note is that information about actual PG concentrations against current intensity was included only once at time $k=1$ $\left(G_{k-1}\right.$, calibration point). However, if more calibration points were used or needed, they would replace the glucose estimation in the previous instant $\left(\hat{G}_{k-1}\right)$ to estimate PG at instant $k$.

In the present study, an exogenous insulin $\left(I_{\mathrm{ex}}\right)$ and glucose infusion $\left(G_{e x}\right)$ were present at some time during the $0-180$-min period: constant insulin infusion in the 30-120-min interval; variable glucose infusion between 30 and $180 \mathrm{~min}$. Therefore, we explored the possibility of including this information in the calibration algorithm as a binary signal (infusion Yes or $\mathrm{No})$ in addition to the other inputs already considered $\left(I_{k}, I_{k-1}\right.$, $\left.\hat{G}_{k-1}\right)$. This is of particular interest because in real-life conditions changes in the conditions of the subject (fed/fasting, insulin bolus/only basal, etc.) may well influence the sensorto-subject relationship, and feeding the calibration algorithm with this information is expected to improve its performance.

It is important to remark that each patient will be represented by one or more LMs to a different extent, depending on its inputs. The shift from one model to another will also be defined by the inputs.

The accuracy of the PG estimations obtained with the new algorithm (with and without the information from binary signals) was calculated using the ISO criteria, ${ }^{24}$ the mean absolute relative difference (MARD), and the median absolute relative difference (M2ARD). Results were compared with those obtained by the GlucoDay, which at the time the study was performed implemented a one-point calibration algorithm: PG estimates $\hat{G}_{k+n}=I_{k+n} \times S$, where $S$ is the sensor sensitivity calculated as $S=I_{k} / G_{k}\left(I_{k}\right.$ and $G_{k}$ are, respectively, the current intensity and the PG concentration at time $k$ ). All data were subjected to repeated-measures analysis of variance with the Huynh-Feldt adjustment for nonsphericity. ${ }^{25}$ The analysis of variance model included the test condition (manufacturer's or different configurations of the new algorithm) as the within-subjects factor. Post hoc comparisons (Newman-Keuls test) were carried out to pinpoint specific differences on significant terms.

\section{Results}

Figure 2 shows PG estimates obtained with the new algorithm, in the configuration not accounting for binary signals, compared with reference PG measurements. On the other hand, the output of each one of the two LMs composing the GM (already weighted by its validity function) can be seen in Figure 3. Figure 2 shows that the estimated signal is quite close to the reference glucose for all patients, indicating good performance of the algorithm even in this case with no exogenous information and only two LMs. Figure 3 shows that each one of the two LMs represents only a subset of the study subjects $\left(\mathrm{LM}_{1}\right.$ for subjects 5 and 6 vs. $\mathrm{LM}_{2}$ for the remaining subjects), whereas only the weighted integration of both LMs to form the GM allows for glucose estimations very close to the actual concentrations (Fig. 2) in the whole population.

Table 1 shows the performance of the proposed algorithm both in its "basic" configuration and considering information from binary signals [i.e., the presence $\left(I_{\mathrm{ex}}, G_{\mathrm{ex}}=1\right)$ or absence $\left(I_{\text {ex }}, G_{\text {ex }}=0\right)$ of insulin and/or glucose infusion]. The comparison with the results from the one-point calibration implemented by the GlucoDay demonstrates that the new calibration algorithm allows for a significant improvement of the accuracy of the glucose estimations. In particular, the inclusion of additional information about the metabolic status further improves the accuracy of glucose estimations, reducing the MARD and M2ARD below $10 \%$. It is noteworthy that the magnitude of improvement was similar for both the whole and the hypoglycemic range. However, likely because of the small sample size, under conditions of hypoglycemia the difference did not reach statistical significance.

\section{Conclusions}

This study describes and validates a new calibration algorithm for CGM systems, based on a dynamic model that includes the relationship between PG levels and measurements in a remote compartment (in this case, the interstitial space). The strength and novelty of this method reside in the structure of the GM, which is composed by several LMs weighted and added together. LMs are defined by a validity function and a linear combination of the inputs, so that each one represents to a variable extent a different (metabolic) condition and/or sensor-subject interaction. Then, each subject will be represented by one or more LMs, and the shift from one LM to another is defined by the inputs (i.e., the output of the sensor but also every other signal containing information relevant to the glucose dynamic). It is noteworthy that each LM has a very simple structure (linear), favoring interpretability of the GM. Moreover, the validity function of LMs $\left(V_{i}\right)$ makes LMs representative of very specific and well-defined regions on the input space, allowing for the identification of the regions they represent. All these may help to elucidate the relationship between the signal(s) from the remote compartment and PG levels, describing different glucose dynamics and sensor behaviors. It does mean that this calibration algorithm can be applied to any sensor that offers some indirect measurement related to PG levels, with the number and parameters of each LM being determined by the particular sensor's output.

When PG is estimated from direct or indirect measurements in any compartment alternative to blood, the following information should be ideally included into the calibration algorithm in order to improve the accuracy of glucose estimations: (1) the intrinsic dynamic of the sensor, (2) glucose dynamic between plasma and the remote compartment, and (3) factors influencing the previous two dynamics. Indeed, sensors of different nature, or even sensors belonging to the same class, may each have a specific intrinsic dynamic. In this regard, recently it has been shown how much of the lag time of continuous glucose sensors is in fact due to the intrinsic delay in the sensor response to changes in glucose concentrations. ${ }^{26}$ Additionally, the interaction between a sensor and the remote compartment of measurement is likely to be specific: in the case of minimally invasive sensors (which to date are the only commercially available) it depends on the biocompatibility of the materials used and on the inflammatory reaction following its insertion. This relationship is complex, may be time-dependent (as the foreign body reaction progresses), and certainly is specific to each individual. ${ }^{27,28}$ 


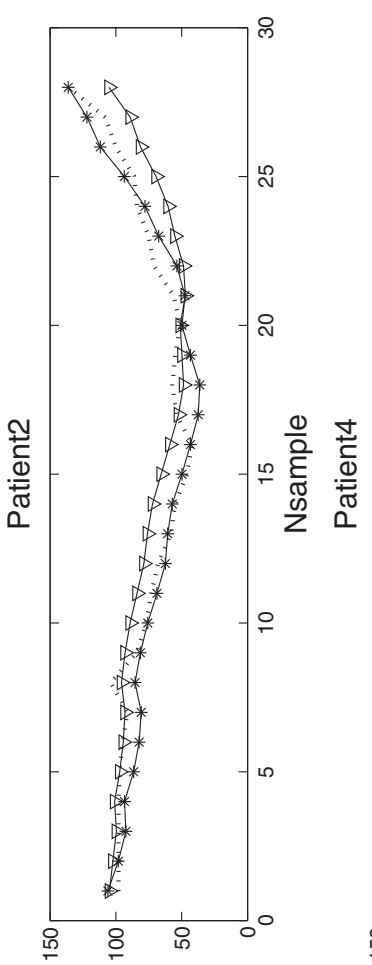

(7p/бu) əsoงnן

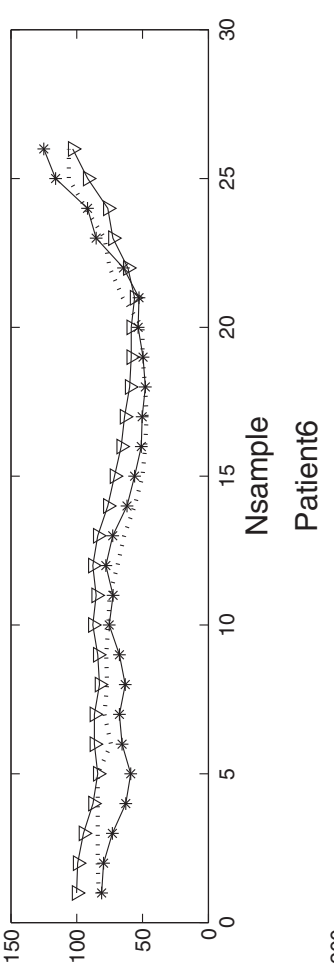

(าp/6u) əsoวnן

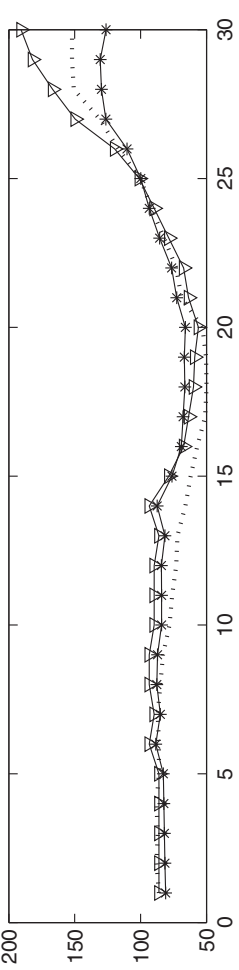

(ך/бu) әsoonן

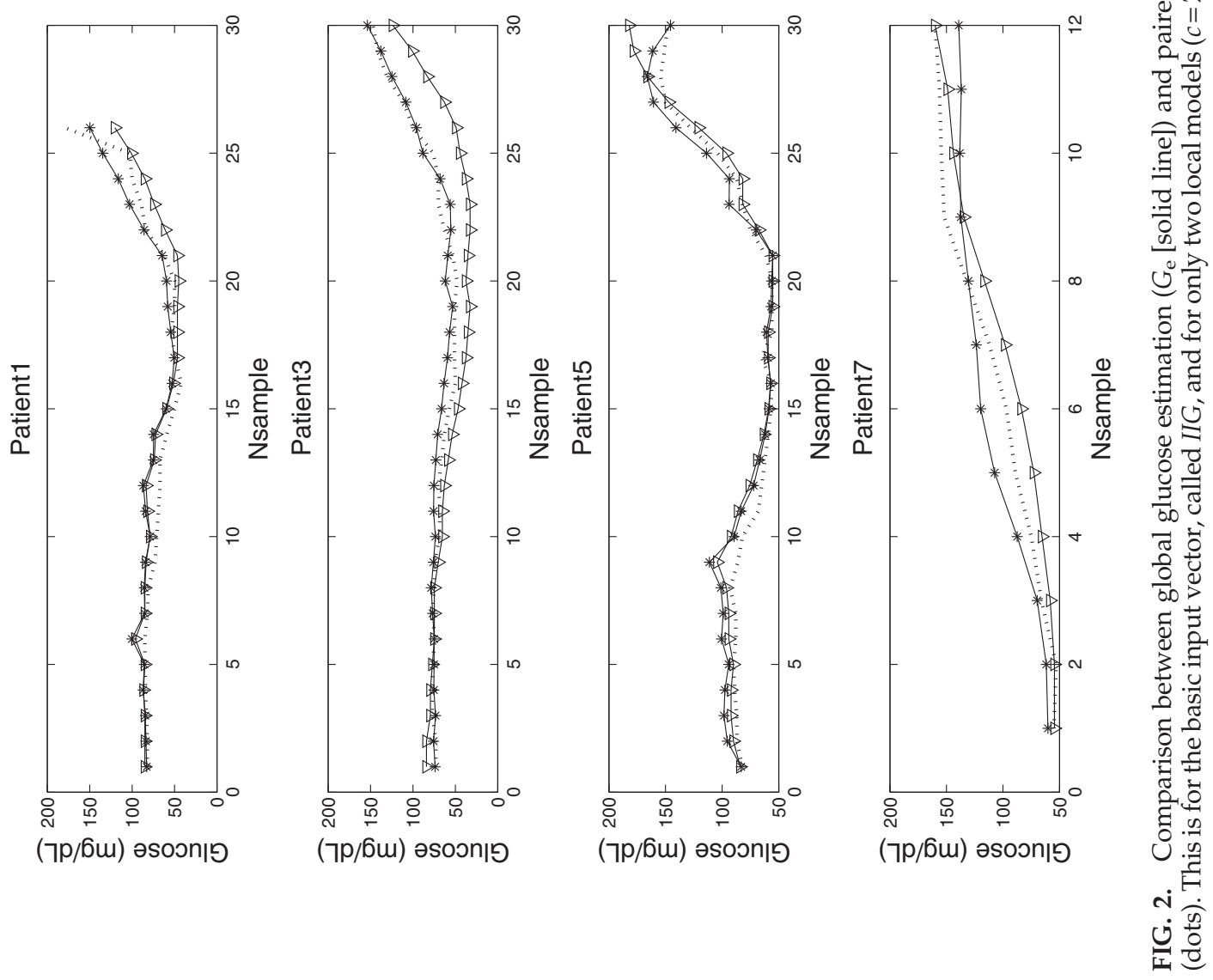




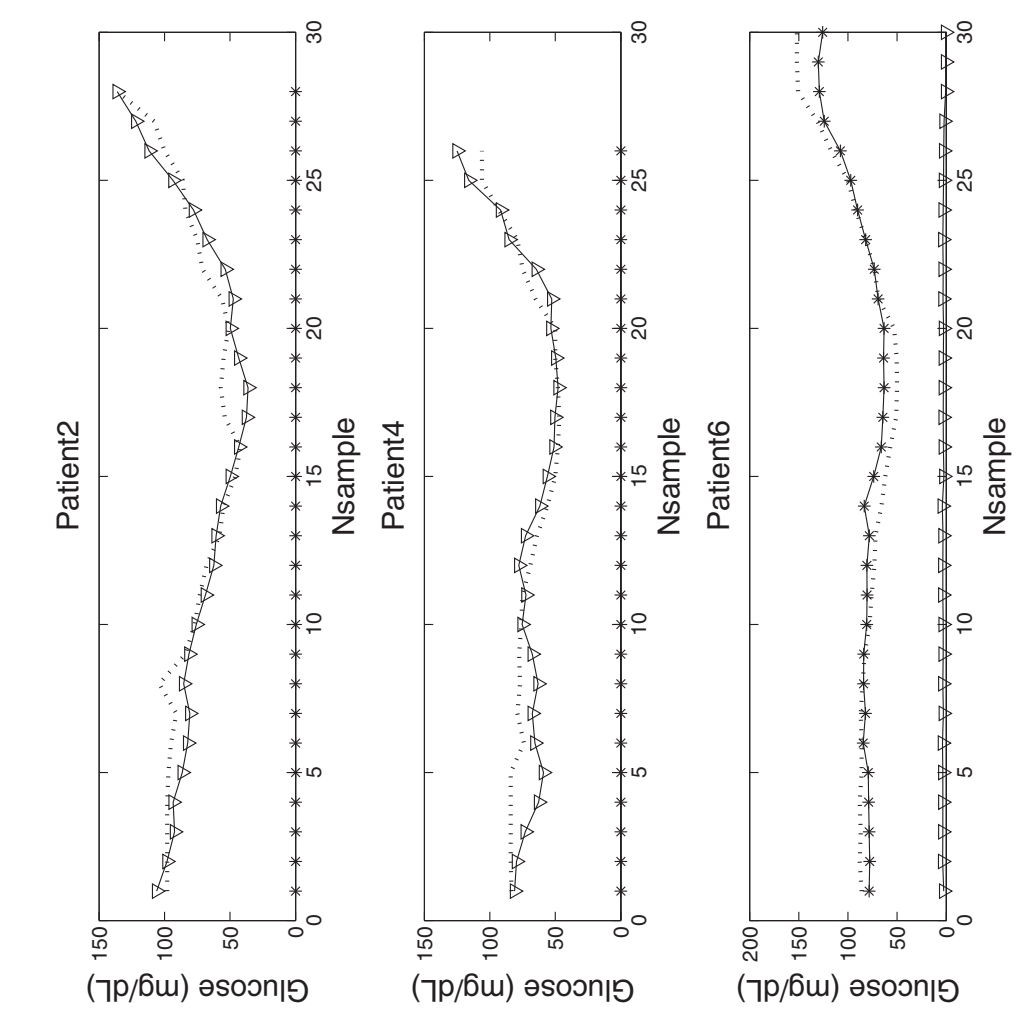

包
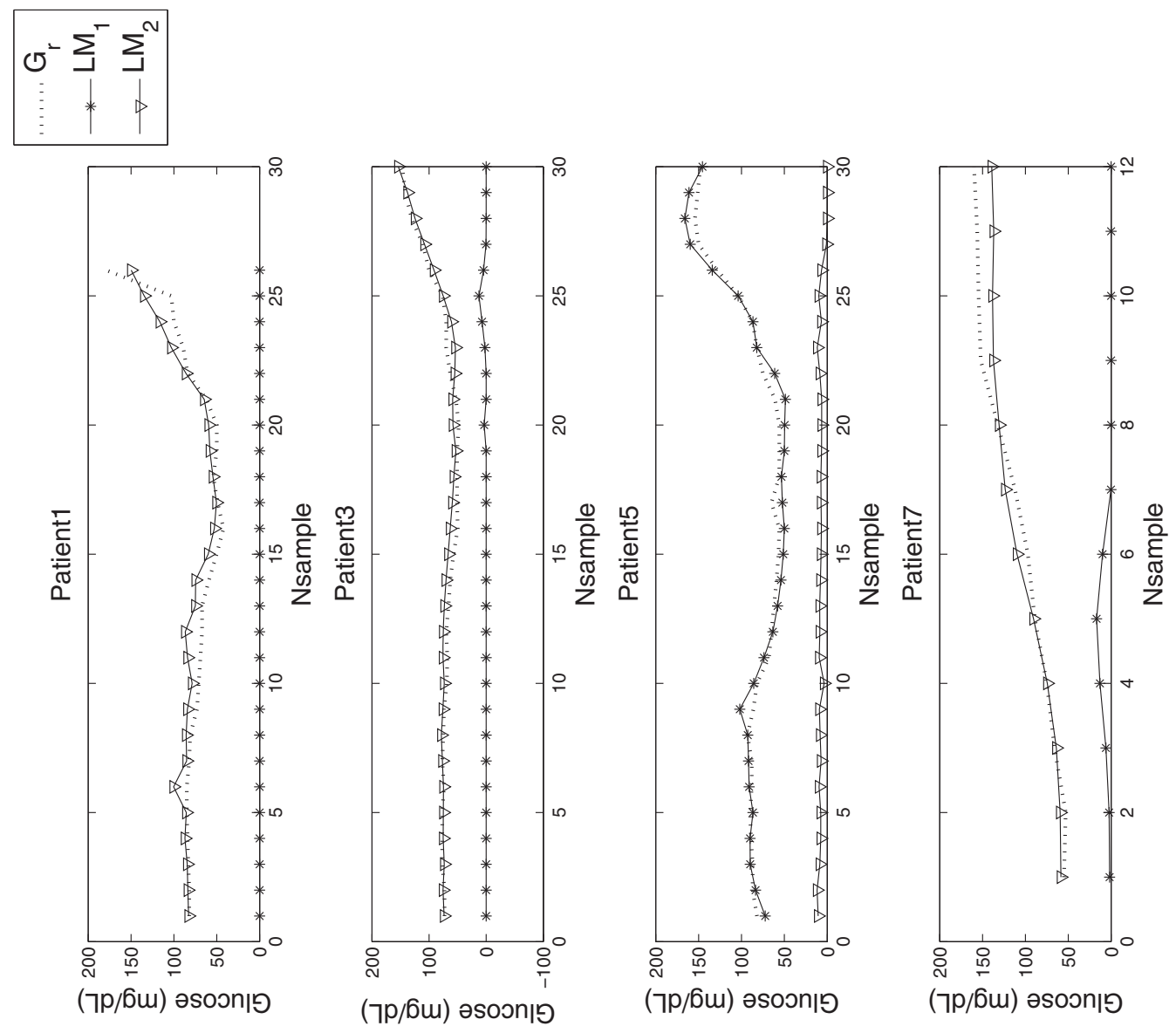

o 0

के

过导导

노요

द्वेन ह

.

ㅇํㄹ 올

I

훙 용

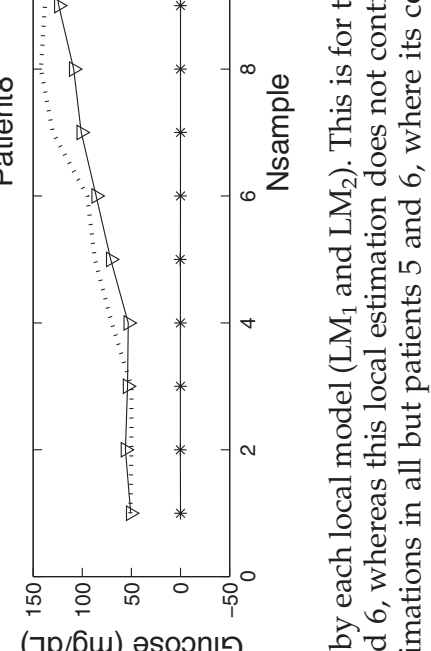

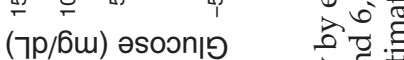

릉 대요

\&

ธี चै

気 है

$500 \frac{\pi}{2}$

क ज

동 है

त

.

के के

ङ

च

वี

잉 0

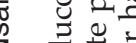

on

릉

흔

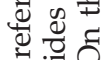

車

过

उ $\sum_{0}$

范牢

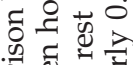

ํํำ

舟

हี

บ శ్ శี

लं士

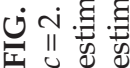




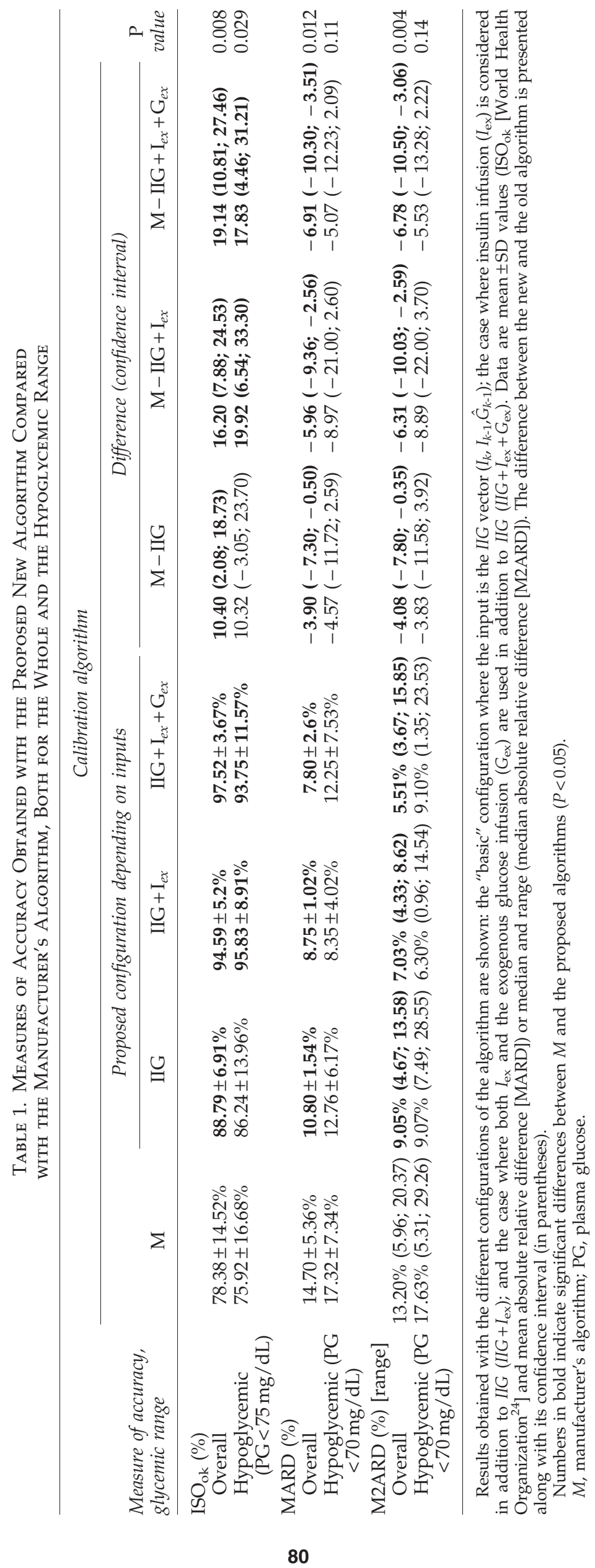


However, to date the issue of enhanced calibration algorithm has received poor attention with only a few scientific contributions. Knobbe and Buckingham ${ }^{16}$ were the first who considered intercompartmental glucose dynamics and developed a five-state extended Kalman filter for the estimation of subcutaneous glucose levels. Facchinetti et al. ${ }^{18}$ further developed the strategy proposed by Knobbe and Buckingham $^{16}$ and proposed the "enhanced Bayesian calibration method" based on an extended Kalman filter estimating interstitial glucose, PG, and sensor sensitivity along time. The method is intended to be used in cascade to any calibration algorithm built in commercial CGM devices and was validated on simulated data representative of diabetes subjects, showing improved CGM accuracy compared with the method of Knobbe and Buckingham. ${ }^{16}$ However, a drawback of this validation is the use of the same model of interstitial glucose and sensor sensitivity for data generation and state estimation, although in the first case a robustness analysis considering discrepancies in lag time estimation is conducted. Furthermore, as the authors acknowledge, application of the enhanced Bayesian calibration method to real data has two main limitations: first, it requires the knowledge of the variances of both state and measurement processes, which in reallife conditions are unknown; second, the existence of a burn-in period, considered as one day by the authors.

Leal et al. ${ }^{29}$ considered sensor and glucose dynamics using a different approach. In particular, autoregressive techniques were applied to CGMS $^{\circledR}$ System Gold ${ }^{\text {TM }}$ (Medtronic, Northridge, $\mathrm{CA}$ ) to monitor data from a clinical study in patients with diabetes. A third-order model of the relationship between current intensity given by the monitor and reference PG measurements was obtained. Predictions given by the model were corrected at every calibration point introduced by the patient, and a cross-validation analysis yielded a substantial improvement of the accuracy of glucose estimations. ${ }^{28} \mathrm{How}-$ ever, a drawback of autoregressive models is that frequent recalibration may be needed to ensure a good performance.

At variance with the previous approaches, the proposed calibration algorithm, thanks to its structure of LMs, is better suited to find and correct for all the above-mentioned dynamics, improving the accuracy of glucose estimations. Results from the present study should be considered as a proof of concept. Indeed, in the small population studied at least two different dynamics of the sensor-to-subject interaction were found, each one detected by a different $L M$ : $\mathrm{LM}_{1}$ for subjects 5 and 6 and $\mathrm{LM}_{2}$ for the other subjects. The reason for the existence of two different LMs was easily explained by analysis of the electric signal from the sensors. Although PG and insulin concentrations were very similar in all subjects (it was a clamp study), there were two "clusters" of current intensity, with the one from sensors inserted in subjects 5 and 6 being much smaller compared with the other. This could be probably due to differences in sensor sensitivity, which were captured automatically by the algorithm. Consideration of both dynamics by means of the two independent LMs allowed for a significant improvement of glucose estimations compared with the original algorithm implemented by the GlucoDay. Additionally, the proposed algorithm admits the introduction of information about the metabolic condition of the subject as a binary signal. This further improved the accuracy of the glucose estimation and appears to be an interesting feature because any information potentially relevant to the sensor-to-subject interaction (physical activity/inactivity, fed/fasting state, etc.) can be used to feed the algorithm.

However, the study has three main limitations. (1) The study population is composed by just eight healthy subjects, and data should be considered preliminary. Thus it is quite probable that the structure of the obtained algorithm is not representative of the general population of people with diabetes. Indeed, getting a calibration algorithm applicable to the general population of people with diabetes would require a specific clinical study in a representative sample, and validation of the obtained algorithm should be carried out in a different study involving larger numbers of subjects with both type 1 and type 2 diabetes. However, in this case the structure of the proposed algorithm is not expected to change regarding the inputs to be considered, although more LMs would probably be needed to cover the greater heterogeneity of local behaviors. (2) Data from reference PG measurement are available for a limited time for each subject. This could in theory have reduced the possibility of identifying different glucose dynamics and sensor behaviors. However, during the clamp study, glucose and insulin concentrations varied through all the clinical significant ranges of eu-, hypo-, and hyperglycemia, giving a good picture of the changes observed in real-life conditions. In contrast, longer studies should be performed to identify changes in the dynamics of the sensor over time, avoiding underestimation of the number $c$ of LMs needed to describe the PG/remote compartment glucose relationship. This is particularly true for minimally invasive sensors where biofouling, inflammation, and foreign body reaction (which are probably tissue and subject specific) induce changes in the sensor's response to variations in glucose concentration. (3) Finally, to prove robustness of the algorithm to cope with variability, the results must be confirmed in a sample representative of the population of patients with diabetes. In this regard, from a phylogenetic point of view it is expected that the inter-subject variations in the intercompartmental glucose dynamics, as well as the spectrum of the inflammatory responses to the sensor's insertion, are limited. However, because of changes in the microcirculation, the variability of the sensor-to-subject interaction (i.e., different sensor sensitivities, sensor's drift overtime, metabolic conditions, etc.) might be greater in patients with diabetes, especially those with microvascular complications. ${ }^{30}$ Nevertheless, the accuracy of CGM in patients with diabetes seems to be not different compared with healthy subjects, indicating that the theoretical greater variability associated with the diabetes condition may have limited practical impact.

Despite its limitations, in this proof-of-concept study the LMs technique is demonstrated to be an effective approach for CGM calibration algorithms. Indeed, aside the very good results obtained in terms of accuracy of glucose estimations, the short computational time associated with this methodology makes it feasible for real-time monitoring and implementation in every glucose sensor.

\section{Acknowledgments}

The authors acknowledge the partial funding of this work by the Spanish Ministry of Science and Innovation projects DPI2007-66728-C02-01 and DPI2010-20764-C02-01 and by the European Union through FEDER funds and grant FP7-PEOPLE-2009-IEF, Reference 252085. F.B.R. is the recipient of a 
fellowship (FPU AP2008-02967) from the Spanish Ministry of Education.

\section{Author Disclosure Statement}

No competing financial interests exist.

\section{References}

1. Goldstein DE, Little RR, Lorenz RA, Malone JI, Nathan DM, Peterson CM: Tests of glycemia in diabetes. Diabetes Care 2004;27(Suppl 1):S91-S93.

2. The Juvenile Diabetes Research Foundation Continuous Glucose Monitoring Study Group: Continuous glucose monitoring and intensive treatment of type 1 diabetes. $\underline{\mathrm{N}}$ Engl J Med 2008;359:1464-1476.

3. Kovatchev B, Anderson S, Heinemann L, Clarke W: Comparison of the numerical and clinical accuracy of four continuous glucose monitors. Diabetes Care 2008;31:1160-1164.

4. Clarke WL, Anderson S, Farhy L, Breton M, Gonder-Frederick L, Cox D, Kovatchev B: Evaluating the clinical accuracy of two continuous glucose sensors using continuous glucose-error grid analysis. Diabetes Care 2005;28:2412-2417.

5. Wentholt IM, Vollebregt MA, Hart AA, Hoekstra JB, DeVries JH: Comparison of a needle-type and a microdialysis continuous glucose monitor in type 1 diabetic patients. Diabetes Care 2005;28:2871-2876.

6. Zisser HJ: Accuracy of the SEVEN continuous glucose monitoring system: comparison with frequently sampled venous glucose measurements. I Diabetes Sci Technol 2009; 3:1146-1154.

7. Keenan DB, Cartaya R, Mastrototaro JJ: Accuracy of a new real-time continuous glucose monitoring algorithm. I Diabetes Sci Technol 2010;4:111-118.

8. Aussedat B, Dupire-Angel M, Gifford R, Klein JC, Wilson GS, Reach G: Interstitial glucose concentration and glycemia: implications for continuous subcutaneous glucose monitoring. Am J Physiol Endocrinol Metab 2000;278:E716-E728.

9. Jansson PA, Fowelin J, Smith U, Lonnroth P: Characterization by microdialysis of intracellular glucose level in subcutaneous tissue in humans. Am J Physiol 1988;255:E218-E220.

10. Thomé-Duret $V$, Reach G, Gangnerau MN, Lemonnier F, Klein JC, Zhang Y, Hu Y, Wilson GS: Use of a subcutaneous glucose sensor to detect decreases in glucose concentration prior to observation in blood. Anal Chem 1996;68:3822-3826.

11. Monsod TP, Flanagan DE, Rife F, Saenz R, Caprio S, Sherwin RS, Tamborlane WV: Do sensor glucose levels accurately predict plasma glucose concentrations during hypoglycemia and hyperinsulinemia? Diabetes Care 2002;25:889-893.

12. Boyne MS, Silver DM, Kaplan J, Saudek CD: Timing of changes in interstitial and venous blood glucose measured with a continuous subcutaneous glucose sensor. Diabetes 2003;52:2790-2794.

13. Sternberg F, Meyerhoff C, Mennel FJ, Mayer H, Bischof F, Pfeiffer EF: Does fall in tissue glucose precede fall in blood glucose? Diabetologia 1996;39:609-612.

14. Rebrin K, Steil GM, van Antwerp WP, Mastrototaro JJ: Subcutaneous glucose predicts plasma glucose independent of insulin: implications for continuous monitoring. Am J Physiol 1999;277:E561-E571.

15. Bequette BW: Continuous glucose monitoring: real-time algorithms for calibration, filtering, and alarms. J Diabetes Sci Technol 2010;4:404-418.
16. Knobbe JE, Buckingham B: The extended Kalman filter for continuous glucose monitoring. Diabetes Technol Ther 2005; 7:15-27.

17. Kuure-Kinsey M, Palerm CC, Bequette BW: A dual-rate Kalman filter for continuous glucose monitoring. Conf Proc IEEE Eng Med Biol Soc 2006;1:63-66.

18. Facchinetti A, Sparacino G, Cobelli C: Enhanced accuracy of continuous glucose monitoring by online extended Kalman filtering. Diabetes Technol Ther 2010;10:353-363.

19. Rossetti P: Estimating plasma glucose from interstitial glucose: the issue of calibration algorithms in commercial continuous glucose monitoring devices. Sensors 2010;10: 10936-10952.

20. Bondia J, Barcelo-Rico F, Diez JL, Rossetti P, Vehi J, Leal YT: Sistema y método de estimación de glucosa en plasma. Spanish Patent application P201130811. May 19, 2011.

21. Rossetti P, Porcellati F, Fanelli CG, Bolli GB: Evaluation of the accuracy of microdialysis-based glucose sensor during insulin-induced hypoglycemia, its recovery, and posthypoglycemic hyperglycemia in humans. Diabetes Technol Ther 2006;8:326-337.

22. Barcelo-Rico F, Diez JL, Bondia J: New possibilistic method for discovering linear local behavior using hyper-Gaussian distributed membership function. Knowledge Information Systems (27 May 2011), pp. 1-27. doi: 10.1007/s10115-011-0385-5.

23. DeCarlo LT: On the meaning and use of kurtosis. Psychol Methods 1997;2:292-307.

24. World Health Organization: ISO 15197:2003. In Vitro Diagnostic Test Systems-Requirements for Blood-Glucose Monitoring Systems for Self-Testing in Managing Diabetes Mellitus; TC/SC: TC 212; ICS: 11.100.10; Stage: 90.92 (200902-16). www.iso.org (accessed June 2011).

25. Winer BJ, Brown DR, Michels KM: Statistical Principles in Experimental Design, $3^{\text {rd }}$ ed. New York: McGraw Hill, 1991: 497-582.

26. Davey RJ, Low C, Jones TW, Fournier PA: Contribution of an intrinsic lag of continuous glucose monitoring systems to differences in measured and actual glucose concentrations changing at variable rates in vitro. I Diabetes Sci Technol 2010;4:1393-1399.

27. Koschwanez HE, Reichert WM: In vitro, in vivo and post explanation testing of glucose-detecting biosensors: current methods and recommendations. Biomaterials 2007;28:36873703.

28. Onuki Y, Bhardwaj U, Papadimitrakopoulos F, Burgess DJ: A review of the biocompatibility of implantable devices: current challenges to overcome foreign body response. I Diabetes Sci Technol 2008;2:1003-1015.

29. Leal YT, Garcia-Gabin W, Bondia J, Esteve E, Ricart W, Fernández-Real JM, Vehí J: Real-time glucose estimation algorithm for continuous glucose monitoring using autoregressive models. J Diabetes Sci Technol 2010;4:391-402.

30. Rosei EA, Rizzoni D: Small artery remodelling in diabetes. J Cell Mol Med 2010;14:1030-1036.

Address correspondence to: Fátima Barceló-Rico, M.Sc.

Institut Universitari d'Automàtica i Informàtica Industrial Universitat Politècnica de València CP 46022, València, Spain

E-mail: fabarri@upv.es 